\title{
Urinary Metabolite Signatures for Predicting Elderly Stroke Survivors with Depression
}

This article was published in the following Dove Press journal:

Neuropsychiatric Disease and Treatment

\author{
Jin Chen ${ }^{1, *}$ \\ Yan-ni Lv ${ }^{2, *}$ \\ Xiao-bing $\mathrm{Li}^{1, *}$ \\ Jia-jun Xiong ${ }^{1} *$ \\ Hui-ting Liang' \\ Liang $\mathrm{Xie}^{3}$ \\ Chen-yi Wan' \\ Yun-qing Chen' \\ Han-sen Wang ${ }^{3}$ \\ Pan $\mathrm{Liu}^{3}$ \\ He-qing zheng ${ }^{3}$ \\ 'Department of Neurology, The First \\ Affiliated Hospital of Nanchang \\ University, Nanchang, Jiangxi, 330006, \\ People's Republic of China; ${ }^{2}$ Department \\ of Pharmacy, The First Affiliated Hospital \\ of Nanchang University, Nanchang, \\ Jiangxi, 330006, People's Republic of \\ China; ${ }^{3}$ Department of Neurology, The \\ Second Affiliated Hospital of Nanchang \\ University, Nanchang, Jiangxi, 330006, \\ People's Republic of China
}

*These authors contributed equally to this work
Correspondence: Jin Chen Department of Neurology, The First Affiliated Hospital of Nanchang University, No. 17 Yongwaizheng Road, Nanchang, Jiangxi, 330006, People's Republic of China

Email jin_chen080706@yeah.net
Background: Post-stroke depression (PSD) is a major complication in stroke survivors, especially in elderly stroke survivors. But there are still no objective methods to diagnose depression in elderly stroke survivors. Thus, this study was conducted to identify potential biomarkers for diagnosing elderly PSD subjects.

Methods: Elderly (60 years or older) stroke survivors with depression were assigned into the PSD group, and elderly stroke survivors without depression and elderly healthy controls (HCs) were assigned into the non-depressed group. Urinary metabolite signatures obtained from gas chromatography-mass spectrometry (GC-MS)-based metabolomic platform were collected. Both univariate and multivariate statistical analysis were used to find the differential urinary metabolites between the two groups.

Results: The 78 elderly HCs, 122 elderly stroke survivors without depression and 124 elderly PSD subjects were included. A set of 13 differential urinary metabolites responsible for distinguishing PSD subjects from non-depressed subjects were found. The Phenylalanine, tyrosine and tryptophan biosynthesis, Phenylalanine metabolism and Galactose metabolism were found to be significantly changed in elderly PSD subjects. The phenylalanine was significantly negatively correlated with age and depressive symptoms. Meanwhile, a biomarker panel consisting of 3-hydroxyphenylacetic acid, tyrosine, phenylalanine, sucrose, palmitic acid, glyceric acid, azelaic acid and $\alpha$-aminobutyric acid was identified.

Conclusion: These results provided candidate molecules for developing objective methods to diagnose depression in elderly stroke survivors, suggested that taking supplements of phenylalanine might be an effective method to prevent depression in elderly stroke survivors, and would be helpful for future revealing the pathophysiological mechanism of PSD.

Keywords: post-stroke depression, metabolomics, biomarker

\section{Introduction}

Post-stroke depression (PSD) is a common neuropsychiatric disorder that can seriously affect the quality of life of stroke survivors, especially the elderly ones. ${ }^{1}$ The incidence rate of PSD is about $30 \%$ at any time up to five years after stroke. ${ }^{2}$ A review reported that the depressive symptoms could slowly rise in first six months after stroke, then slightly alleviate at 12 months, and worsen again in the next year. ${ }^{3}$ Epidemiological study showed that PSD might interfere with stroke recovery. ${ }^{4}$ Because it could decrease the will of patients to participate in rehabilitation programs, and finally result in a longer length of hospital stay. ${ }^{5}$ Moreover, PSD could seriously affect the neural functional recovery of patients and the depressed patients have fewer daily activities. This might lead to the increased mortality and disability rate during the 2-5 years after stroke. ${ }^{6}$ Therefore, it is very important to early diagnose and treat PSD. 
Although other researchers and we have done many works, the treatment and diagnosis of depression is still in the exploratory stage. ${ }^{7-10}$ The diagnosis of depression in stroke survivors is still based on the subjective identification of symptom clusters, which often causes a misdiagnosis or under-diagnosis. ${ }^{11}$ To solve this problem, many works have been done to identify PSDrelated biomarkers. ${ }^{12,13}$ Zhao et al found that the serum neurofilament light could be used to make early diagnoses of PSD. ${ }^{12}$ Another study suggested that MiR-140-5p might be closely related with the pathogenesis of PSD and could be used as a novel early warning biomarker for diagnosing PSD. ${ }^{13}$

Some studies have identified several urinary biomarkers for PSD. ${ }^{14-16}$ But none of these previous studies have taken age-based differences into consideration, especially considering that elderly stroke survivors are more likely to be depressed. ${ }^{1}$ In recent decades, metabolomics is widely used to find potential biomarkers for many diseases. ${ }^{17,18}$ Using metabolomic approaches, researchers have identified some potential urinary biomarkers that could be used to diagnose patients with depression. ${ }^{19,20}$ Here, we collected urinary metabolomic data from gas chromatography-mass spectroscopy (GC-MS) based metabonomic platform. The purpose of this study was to find the differential urinary metabolites in elderly PSD subjects and identify potential biomarkers for objectively diagnosing depression in elderly stroke survivors.

\section{Patients and Methods}

\section{Subjects Obtained}

This study was approved by the Ethical Committee of the First Affiliated Hospital of Nanchang University (NCU20180219) and conducted in accordance with the Declaration of Helsinki. The diagnostic criteria for ischemic or hemorrhagic stroke (confirmed by the fourth National Conference on Cerebrovascular Diseases) were used to diagnose stroke. PSD patients were screened by experienced psychiatrists using the DSM-IV, and the Hamilton Depression Rating Scale (HDRS) score was used to assess the depressive symptoms of stroke survivors. The elderly (age $\geq 60$ ) stroke survivors with HDRS score $\geq 17$ were assigned into the PSD group, and others were assigned into the non-depressed group. The elderly stroke survivors were excluded if they had illicit drug use, alcohol abuse, preexisting mental disorders before stroke, or systemic medical illness. Meanwhile, the elderly (age $\geq 60$ ) healthy controls (HCs) were also recruited. Finally, 78 elderly HCs and 122 elderly stroke survivors without depression were included and assigned into the non-depressed group, and 124 elderly stroke survivors with depression were included and assigned into the PSD group. The included subjects provided the written informed consents before sample collection. The detailed information is described in Table 1.

\section{Sample Processing and Grouping}

The morning urine samples were collected using a sterile cup after 12-hours fasting. The midstream urine was collected. After centrifugation $(1500 \mathrm{~g}$, ten minutes), we divided the obtained supernatant into equal aliquots, and stored them at $-80^{\circ} \mathrm{C}$ until later analysis. The procedure of GC-MS analysis and samples was extracted from the previous studies. ${ }^{14,15}$ The global metabolome analysis was used here. In total, 59 urinary metabolites were analyzed, and the analysis was done in Nanchang University. We randomly assigned the included subjects into training set (44 elderly HCs, 86 elderly stroke survivors without depression and 82 elderly PSD subjects) and testing set (34 elderly HCs, 36 elderly stroke survivors without depression and 42 elderly PSD subjects). Generally

Table I Detailed Information of the Elderly Recruited Subjects

\begin{tabular}{|c|c|c|c|c|c|c|c|c|}
\hline \multirow[t]{2}{*}{ Variables } & \multicolumn{4}{|c|}{ Training Set } & \multicolumn{4}{|l|}{ Testing Set } \\
\hline & HCs & Strokes & PSD & P-value & HCs & Strokes & PSD & P-value \\
\hline Number & 44 & 86 & 82 & - & 34 & 36 & 42 & - \\
\hline Age & 65.20 (3.7I) & $66.88(4.84)$ & $67.22(4.69)$ & $0.49^{\mathrm{a}}$ & $65.41(2.74)$ & $67.19(5.42)$ & $66.17(4.34)$ & $0.99^{\mathrm{a}}$ \\
\hline BMI & $21.68(2.59)$ & $23.13(2.80)$ & $23.47(2.99)$ & $0.12^{\mathrm{a}}$ & $21.98(3.00)$ & $22.37(2.60)$ & $23.73(3.13)$ & $0.20^{\mathrm{a}}$ \\
\hline $\operatorname{Sex}(M / F)$ & $18 / 26$ & $26 / 40$ & $39 / 43$ & $0.57^{\mathrm{b}}$ & $17 / 17$ & $|5 / 2|$ & $21 / 21$ & $0.71^{b}$ \\
\hline HDRS & $0.25(0.6 \mathrm{I})$ & $1.60(1.85)$ & $19.99(5.42)$ & $<0.00001^{\mathrm{a}}$ & $0.59(1.05)$ & $1.67(1.56)$ & $20.8 \mid(5.69)$ & $<0.00001^{a}$ \\
\hline
\end{tabular}

Notes: ${ }^{\text {a }}$-value was obtained from one-way ANOVA test. ${ }^{\mathrm{b}} \mathrm{P}$-value was obtained from Chi-square test.

Abbreviations: M, male; F, female; HCs, healthy controls; BMI, body mass index; PSD, post-stroke depression; HDRS, Hamilton Depression Rating Scale. 
speaking, it was very important to using testing set to validate the results obtained from the training set.

\section{Statistical Analysis}

The Student's $t$-test, non-parametric Mann-Whitney $U$-test, one-way ANOVA test or Chi-square test was conducted when appropriate. The Benjamini-Hochberg false discovery rate was used to correct the obtained p-value. A corrected p-value $<0.05$ was considered to be statistically significant. To alleviate the potential effects of urine samples from the different subjects, the creatinine was used to normalize the raw metabolomics data; meanwhile, to alleviate the potential effects of different orders of magnitude in urinary metabolites, the zero-mean and unitvariance was used to further scale the metabolomics data. $^{20}$

The orthogonal partial least-squares discriminant analysis (OPLS-DA) was a supervised multivariate approach, which is widely used to analyze the Omics data to visualize discrimination between different groups. ${ }^{16,20}$ It was an excellent tool to identify "What's the difference" between the two groups. Therefore, in this study, to find out the metabolic differences between elderly PSD subjects and elderly non-depressed subjects, the OPLS-DA model was firstly built using the training set. Then, the 399-iteration permutation test was conducted to evaluate the goodness-offit of the built OPLS-DA model. The testing set was further used to assess the predicted accuracy of the built model. Finally, the coefficient loading plot of the built OPLS-DA model was used to identify the metabolic differences in elderly PSD subjects. In this study, according to the number of samples in training set, we selected the correlation coefficient of $|r|>0.217$ (equivalent to $p$-value $<0.05$ ) as the cut-off value for identifying the differential urinary metabolites.

To obtain a simplified metabolite biomarker panel, the step-wise logistic-regression analysis was further used to analyze the obtained differential urinary metabolites. Based on Akaike's information criterion (AIC) rule, a best model with balance between accuracy and complexity was obtained. The AIC rule would estimate the quality of each model composed of differential urinary metabolites. It could simultaneously consider the simplicity and the goodness of fit of each model. The best model was the one with minimum AIC value. Then, the receiver-operating characteristic (ROC) curve analysis was conducted to evaluate the diagnostic performance of the obtained biomarker panel. ${ }^{21,22}$ The area under the curve (AUC) was the evaluation index. In addition, we used the Pearson correlation coefficient to assess the relationship between the identified differential urinary metabolites and demographic data (HDRS, BMI and age). To further explore the biological functions of the differential urinary metabolites closely involved in, the online software MetaboAnalyst 4.0 was used here. ${ }^{23}$ IBM SPSS statistical software (Version 19.0, IBM Corp., Armonk, NY) was used for data analysis.

\section{Results}

\section{Different Metabolic Profiling Between Two Groups}

We firstly used the training set to build the OPLS-DA model. To avoid the potential influences of demographic data on our findings, the HDRS, BMI, gender and age were used as covariates here. As shown in Figure 1A, the elderly PSD subjects were intelligibly separated from the elderly nondepressed subjects with little overlap (Figure 1A): 78 of the 82 elderly PSD subjects and 118 of the 130 elderly nondepressed subjects were correctly distinguished. The OPLSDA model was evaluated by the parameters of R2Y and Q2. The values of $\mathrm{R} 2 \mathrm{Y}=0.59$ and $\mathrm{Q} 2=0.45$ demonstrated that the model was reliable. The 399-iteration permutation test indicated that the model was not over-fitted. Meanwhile, the T-predicted scatter plot showed that most of samples from the testing set could be correctly predicted (Figure 1B): 35 of the 42 elderly PSD subjects and 62 of the 70 elderly nondepressed subjects were correctly predicted. These results suggested that there were pronounced metabolic differences between elderly PSD subjects and elderly non-depressed subjects at an overview level.

\section{Differential Urinary Metabolites in Elderly PSD Subjects}

Differential urinary metabolites in elderly PSD subjects were filtrated by the correlation coefficient $(|\mathrm{r}|>0.217)$. In total, 13 identified metabolites with $|r|>0.217$ were identified according to the coefficient loading plots of OPLS-DA model. Compared to elderly non-depressed subjects, the elderly PSD subjects were characterized by higher levels of palmitic acid, azelaic acid, sucrose, glyceric acid, $\alpha$-aminobutyric acid, lactic acid and fructose, along with lower levels of 3-hydroxyisobutyric acid, sorbitol, indoxyl sulphate, 3-hydroxyphenylacetic acid, phenylalanine and tyrosine. Meanwhile, univariate statistical analysis (nonparametric Mann-Whitney $U$-test and Benjamini-Hochberg false discovery rate) was applied to validate the metabolic changes identified by multivariate statistical analysis (OPLS-DA model); 12 of 13 

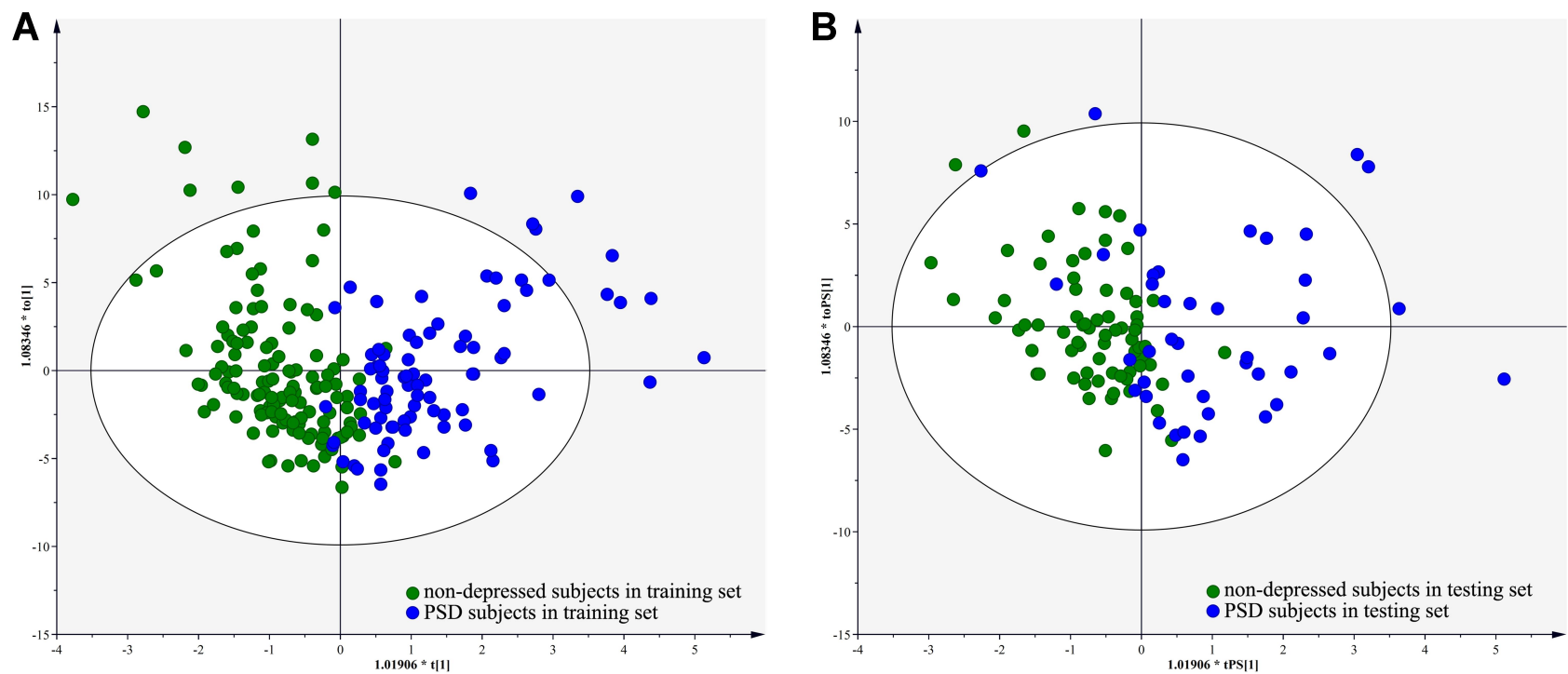

Figure I Metabolomic analysis of urine samples from different groups: (A) OPLS-DA model built with training set showed that the elderly PSD subjects (blue dot) could be intelligibly separated from the elderly non-depressed subjects (green dot); (B) T-predicted scatter plot showed that the model could effectively predict the elderly PSD subjects (blue dot) and elderly non-depressed subjects (green dot) from the testing set.

differential urinary metabolites remained significantly changed (Table 2).

\section{Potential Biomarker Panel for Diagnosing Elderly PSD}

The step-wise logistic-regression analysis based on AIC rule was used to further analyze the identified 13 differential urinary metabolites. The results showed that the most significant deviations between elderly PSD subjects and elderly non-depressed subjects could be described by the following eight differential urinary metabolites: 3-hydroxyphenylacetic acid, tyrosine, phenylalanine, sucrose, palmitic acid, glyceric acid, azelaic acid and $\alpha$ aminobutyric acid (Figure 2). Then, the ROC analysis was used to evaluate the diagnostic performance of the potential biomarker panel consisting of these eight metabolites. The results suggested that this panel could effectively separate elderly PSD subjects from elderly non-depressed subjects with an AUC of 0.9421 in the training set and an AUC of 0.8687 in the testing set (Figure 3 ).

\section{Correlation Analysis and Biological Interpretation}

Pearson correlation analysis showed that there was a significantly positive correlation between BMI and age; there was a significantly negative correlation between age and phenylalanine; there were significantly negative correlations between BMI and five differential urinary metabolites (3-hydroxyisobutyric acid, sorbitol, phenylalanine, tyrosine and azelaic acid); and there were significantly negative correlations between HDRS score and three differential urinary metabolites (phenylalanine, 3-hydroxyisobutyric acid and sucrose). In addition, we used online software MetaboAnalyst 4.0 to conduct pathway analysis, and found that three metabolic pathways were significantly affected in elderly PSD subjects (Figure 4A): Phenylalanine, tyrosine and tryptophan biosynthesis $(p=0.0003$, impact $=1.0)$ (differential urinary metabolites: phenylalanine and tyrosine), Phenylalanine metabolism $(p=0.002$, impact $=0.35)$ (differential urinary metabolites: phenylalanine and tyrosine), and Galactose metabolism $(p=0.017$, impact $=0.039)$ (differential urinary metabolites: sucrose and sorbitol). The detailed information of these three pathways is presented in Figure 4B.

\section{Discussion}

Through a GC-MS metabolomic approach coupled with OPLS-DA model, we identified a set of 13 differential urinary metabolites that could clearly separate elderly PSD subjects from elderly non-depressed subjects. Six differential urinary metabolites (palmitic acid, azelaic acid, sucrose, glyceric acid, fructose and sorbitol) were closely related with energy metabolism, which might indicate the disordered energy balance in elderly PSD subjects. Meanwhile, a potential biomarker panel consisting of eight 
Table 2 Important Metabolites Responsible for Diagnosing Elderly PSD Patients

\begin{tabular}{|c|c|c|c|c|}
\hline Metabolites & p-value ${ }^{a}$ & Adjust p-value $^{b}$ & $\mathbf{R}^{\mathbf{c}}$ & Fold Change $^{d}$ \\
\hline Palmitic acid & 8.IIE-06 & 9.57E-05 & 0.456159 & 0.82 \\
\hline Azelaic acid & 8.95E-07 & I.76E-05 & 0.450246 & 1.29 \\
\hline Sucrose & $2.4 I E-06$ & $3.56 \mathrm{E}-05$ & 0.372082 & 0.97 \\
\hline Glyceric acid & 4.09E-05 & $4.02 \mathrm{E}-04$ & 0.366423 & 1.10 \\
\hline$\alpha$-aminobutyric acid & $9.63 \mathrm{E}-05$ & 7.10E-04 & 0.352394 & 0.64 \\
\hline Lactic acid & 7.37E-07 & 2.17E-05 & 0.284821 & 0.74 \\
\hline Fructose & I.06E-04 & 6.97E-04 & 0.217637 & 0.77 \\
\hline 3-hydroxyisobutyric acid & I.98E-03 & 8.35E-03 & -0.271174 & -0.22 \\
\hline Sorbitol & $8.00 \mathrm{E}-02$ & I.52E-0| & $-0.30 I 555$ & -0.29 \\
\hline 3-hydroxyphenylacetic acid & I.52E-02 & $4.73 \mathrm{E}-02$ & -0.33488 & -0.60 \\
\hline Indoxyl sulphate & $6.18 \mathrm{E}-03$ & 2. $14 \mathrm{E}-02$ & -0.355926 & -0.30 \\
\hline Phenylalanine & I.37E-03 & $6.24 \mathrm{E}-03$ & -0.413967 & -0.40 \\
\hline Tyrosine & I.96E-07 & I.16E-05 & -0.463675 & -0.52 \\
\hline
\end{tabular}

Notes: ${ }^{a} \mathrm{p}$-values obtained from nonparametric Mann-Whitney U-test. ${ }^{b}$ Adjust $\mathrm{p}$-values obtained using Benjamini-Hochberg false discovery rate. ${ }^{\mathrm{c}}$ Correlation coefficient obtained from OPLS-DA model, positive and negative values indicated higher and lower levels in elderly PSD patients, respectively. ${ }^{\mathrm{d} L o g} 2$ fold-change, positive and negative values indicated higher and lower levels in elderly PSD patients, respectively.

differential urinary metabolites was identified. This panel was capable of discriminating the training samples and test samples with AUCs of 0.9421 and 0.8687 , respectively. These results demonstrated that the urinary metabolites held the promise as an objective method to diagnose elderly PSD subjects, and this panel with excellent diagnostic performance in diagnosing depression in elderly stroke survivors should be further explored.

Zheng et al used GC-MS based metabolomic approach to identify some urinary metabolite biomarkers for
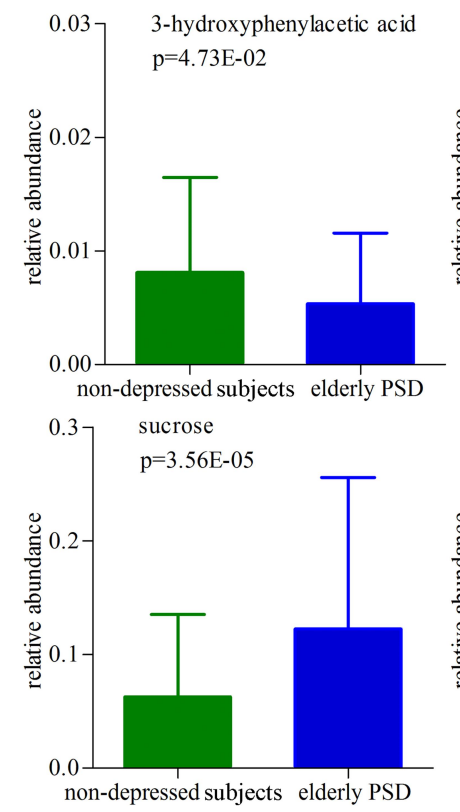
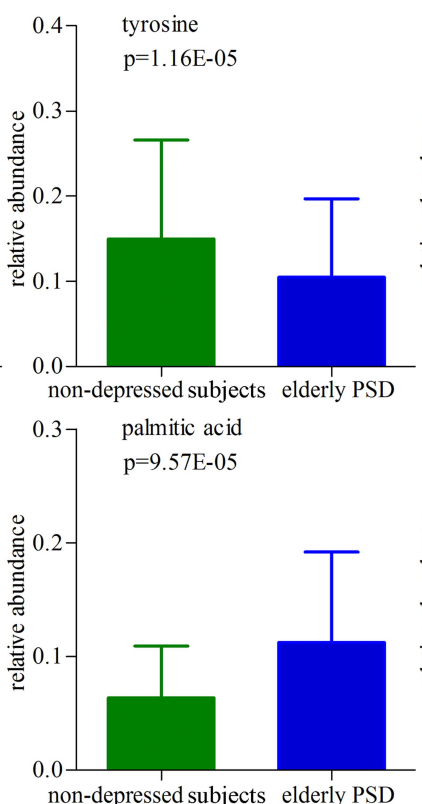
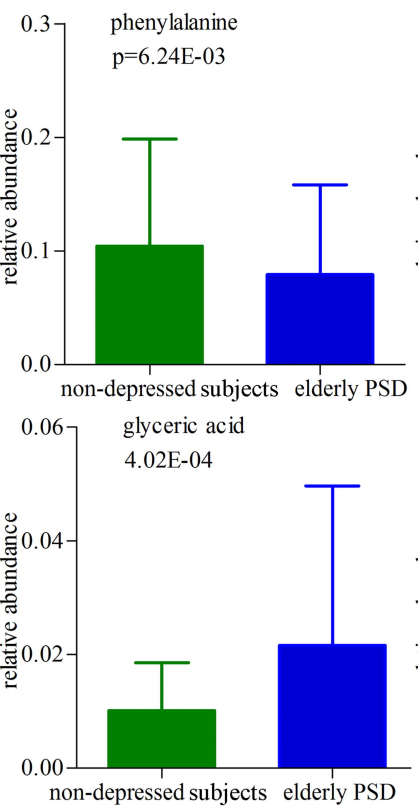

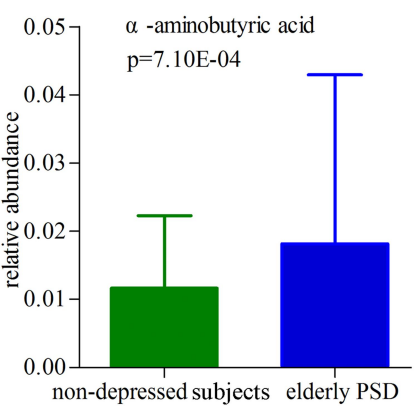

$0.03 \_\quad$ azelaic acid $\mathrm{p}=1.76 \mathrm{E}-05$

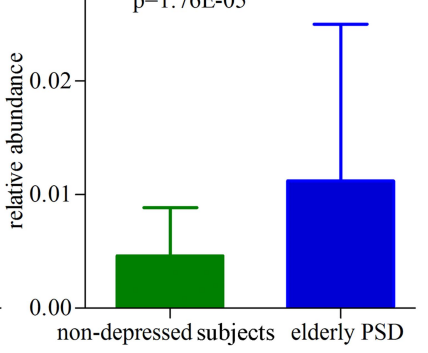

Figure 2 Relative concentrations of these eight urinary metabolite biomarkers for elderly PSD (mean \pm standard deviation). 

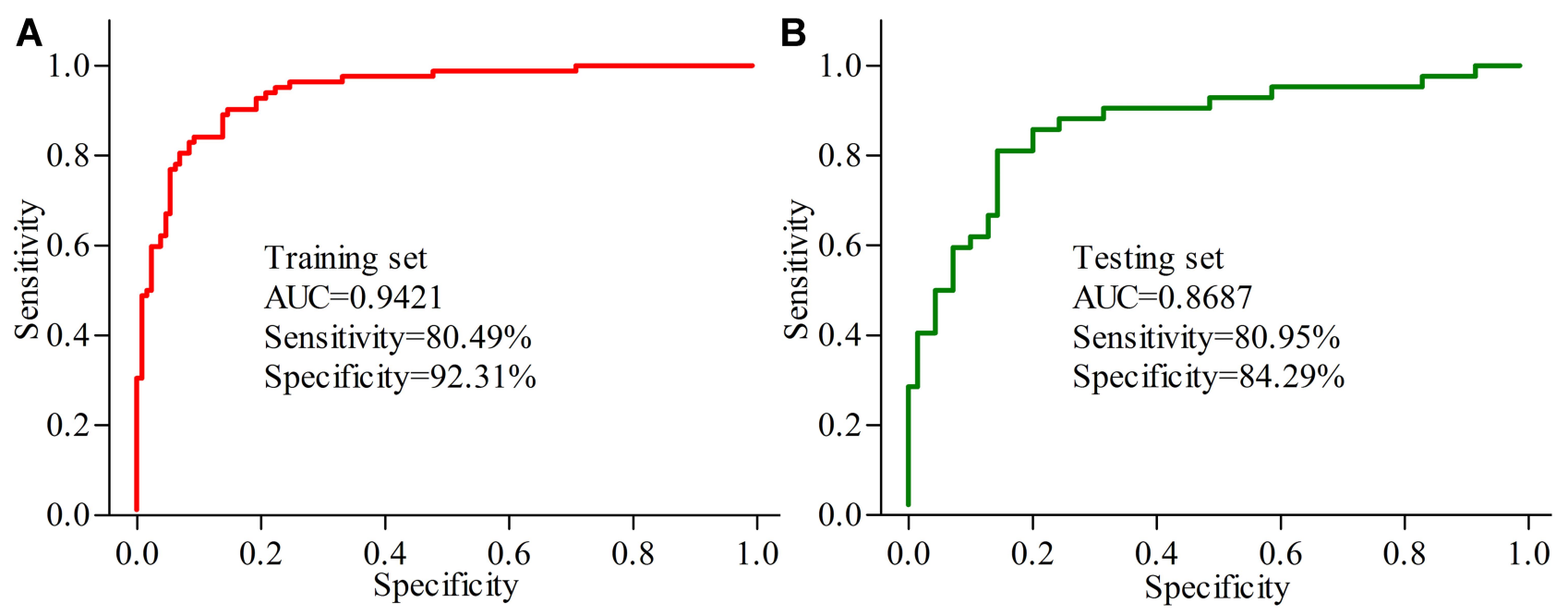

Figure 3 Diagnostic performance of potential biomarker panel for elderly PSD: (A) AUC value in the training set; (B) AUC value in the testing set.

A

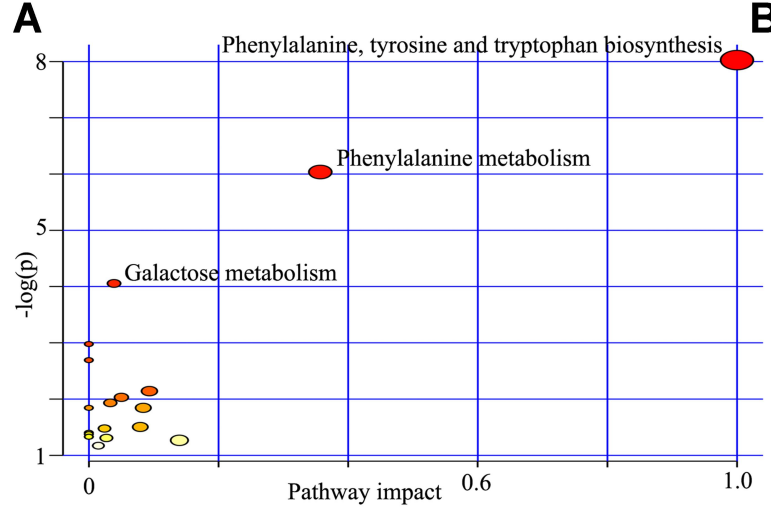

B

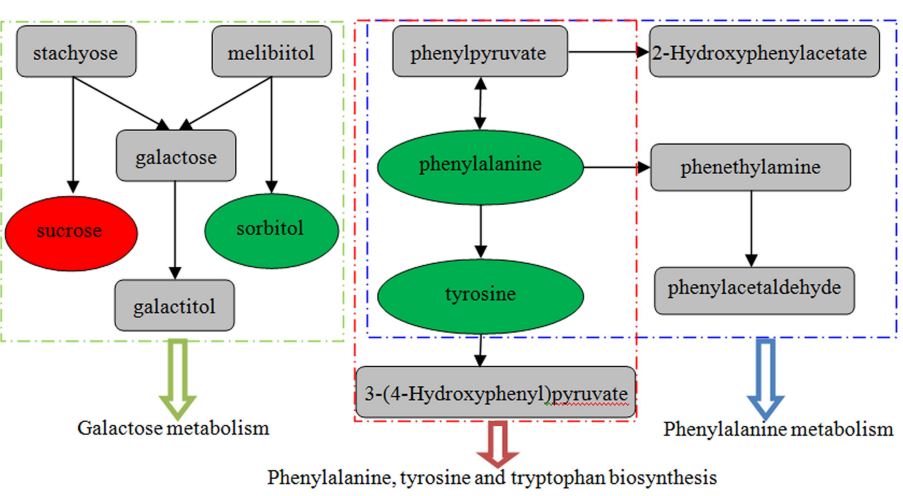

Figure 4 Pathway analysis using the differential urinary metabolites: (A) three metabolic pathways were found to be significantly affected in elderly PSD subjects; (B) the main metabolites involved in these pathways. Red and green ellipses represent the significantly increased and decreased metabolites, respectively, identified in this study.

diagnosing depression. ${ }^{24}$ But these potential biomarkers might not be appropriate for diagnosing depression in stroke survivors. Therefore, to obtain more accurate potential biomarkers for PSD, researchers have done many wonderful works to successfully identify several differential urinary metabolites. ${ }^{14,15}$ Meanwhile, Xie et al identified some potential metabolite biomarkers for middle-aged PSD subjects. ${ }^{16}$ However, these identified potential biomarkers could not effectively separate elderly PSD subjects from elderly non-depressed subjects. Here, we successfully identified a biomarker panel that could effectively diagnose elderly PSD subjects. In addition, it may be meaningful to explore the possibility of combining biomarkers and demographic data; if successful, it may be helpful for developing a more personalized diagnostic method for stroke patients with depression.

The mechanism of PSD remains unclear, which brings a big challenge to its prevention and treatment. Our previous studies found that the disturbance of lipid metabolism and energy metabolism might be closely related to the onset of depression. ${ }^{25-27}$ The neurotransmitter imbalance theory believes that the brain damage resulting from stroke could finally cause the reduced bioavailability of norepinephrine, dopamine and serotonin. ${ }^{28}$ During PSD, there is an obvious increase in numerous pro-inflammatory cytokines and inflammatory markers, such as interferon (INF)$\alpha$ and interleukin (IL)- $1 \beta .{ }^{29}$ Previous studies also reported that there was a close relationship between PSD and the 
functional polymorphism of serotonin transporter genelinked promoter region. ${ }^{30}$ Meanwhile, most of current evidences indicate that the specific location of a lesion may have a critical role in the pathogenesis of PSD. ${ }^{31}$

In general, neurotransmitters, gene polymorphism, inflammatory cytokines and lesion locations may be the four major biological causes of PSD, but none of these theories has been universally accepted. In this study, we found that three differential urinary metabolites (phenylalanine, tyrosine, indoxyl sulphate) in elderly PSD subjects were the metabolic byproducts of gut microbiota. Meanwhile, the association between gut microbiota and depression has become a hotspot in recent decade. ${ }^{32,33}$ Previous study found that there were age-related changes in gut microbiota in patients with depression. ${ }^{34}$ Therefore, the disturbance of gut microbiota might be related with the development of elderly PSD.

Two phenylalanine metabolism-related pathways (Phenylalanine metabolism, Phenylalanine, tyrosine and tryptophan biosynthesis) were found here to be significantly affected in elderly PSD subjects. In addition, phenylalanine was significantly negatively correlated with age, which suggested that the elderly subjects had the lower level of phenylalanine. Moreover, we also found the significantly negative correlation between phenylalanine and HDRS score. It suggested that the subjects with lower level of phenylalanine had the higher HDRS score or more serious depressive symptoms. The stroke could occur at any age, but most cases of stroke still occur in the elderly subjects. ${ }^{35}$ Phenylalanine, as one of the eight essential amino acids, might have the potential ability to relieve pain and depression. Taken together, these results suggested that phenylalanine might be a potential target for preventing and treating depression in elderly stroke survivors.

Limitations should be mentioned here. Firstly, we only adjusted the HDRS score, age, BMI and sex to avoid the potential influences of socio-demographic data on our findings; the effects of other sociodemographic variables, such as marital status and levels of education, were needed future studies to assess. Secondly, all the included stroke survivors received medications to treat stroke; because the medications might affect the metabolomics profiles, ${ }^{36}$ further researches were needed to support and validate our conclusions. Thirdly, the antidepressive effects of taking supplements of phenylalanine were not validated here; future studies should further explore whether taking supplements of phenylalanine can prevent depression in stroke survivors. Fourthly, we failed to confirm the specific metabolic activity of some enzymes in PSD patients according to the changes of differential urinary metabolites from the same metabolic pathway; this point was needed in future studies to further investigate. Fifthly, we enrolled combination of ischemic and hemorrhagic stroke patients, but patients with different kinds of stroke likely have different urinary metabolic profiles; thus, future studies should validate whether our conclusion was appropriate for any single kind of stroke.

In conclusion, through analyzing the urinary metabolites in elderly PSD subjects, a set of 13 differential urinary metabolites responsible for the discrimination between elderly PSD subjects and elderly non-depressed subjects were identified. Meanwhile, a potential biomarker panel consisting of eight differential urinary metabolites was found to be effective in diagnosing elderly PSD subjects. Our results would be helpful for future developing an objective diagnostic method for elderly PSD, and provide novel insights into the mechanism of PSD.

\section{Acknowledgments}

This work was supported by the Key project of Education Department of Jiangxi Province (grant no.GJJ170029) and National Natural Science Foundation of China (grant nos. 31860276). The funders had no role in study design, data collection and analysis, decision to publish, or preparation of the manuscript.

\section{Disclosure}

The authors declare no financial or other conflicts of interest.

\section{References}

1. Rogers S. Poststroke depression screening: an executive summary. J Neurosci Nurs. 2017;49(2):66-68. doi:10.1097/ JNN.0000000000000270

2. Lin F-H, Yih DN, Shih F-M, et al. Effect of social support and health education on depression scale scores of chronic stroke patients. Medicine (Baltimore). 2019;98(44):e17667. doi:10.1097/ MD.0000000000017667

3. Werheid K. A two-phase pathogenic model of depression after stroke. Gerontology. 2016;62:33-39. doi:10.1159/000381876

4. Ojagbemi A, Akinyemi R, Baiyewu O. Cognitive dysfunction and functional limitations are associated with major depression in stroke survivors attending rehabilitation in Nigeria. NeuroRehabilitation. 2014;34(3):455-461. doi:10.3233/NRE-141061

5. Sugawara N, Metoki N, Hagii J, et al. Effect of depressive symptoms on the length of hospital stay among patients hospitalized for acute stroke in Japan. Neuropsychiatr Dis Treat. 2015;11:2551-2556. doi:10.2147/NDT.S91303 
6. Bartoli F, Lillia N, Lax A, et al. Depression after stroke and risk of mortality: a systematic review and meta-analysis. Stroke Res Treat. 2013;2013:862978. doi:10.1155/2013/862978

7. Wu M, Tian T, Mao Q, et al. Associations between disordered gut microbiota and changes of neurotransmitters and short-chain fatty acids in depressed mice. Transl Psychiatry. 2020;10(1):350. doi:10.1038/s41398-020-01038-3

8. Liao D, Chen Y, Guo Y, et al. Salvianolic acid B improves chronic mild stress-induced depressive behaviors in rats: involvement of AMPK/SIRT1 signaling pathway. J Inflamm Res. 2020;13:195-206. doi:10.2147/JIR.S249363

9. Yang Y, Chen J, Liu C, et al. The extrinsic coagulation pathway: a biomarker for suicidal behavior in major depressive disorder. $\mathrm{Sci}$ Rep. 2016;6(1):32882. doi:10.1038/srep32882

10. Wu D, Peng Y, Zhou J, et al. Identification and validation of argininosuccinate synthase as a candidate urinary biomarker for major depressive disorder. Clin Chim Acta. 2015;451(Pt B):142-148. doi:10.1016/j.cca.2015.09.018

11. Chen L-S, Eaton WW, Gallo JJ, Nestadt G. Understanding the heterogeneity of depression through the triad of symptoms, course and risk factors: a longitudinal, population-based study. J Affect Disord. 2000;59(1):1-11. doi:10.1016/S0165-0327(99)00132-9

12. Zhao H, Mo M, Miao C, et al. Association of serum biomarker neurofilament light concentration with post-stroke depression: a preliminary study. Gen Hosp Psychiatry. 2020;64:17-25. doi:10.1016/j.genhosppsych.2020.01.006

13. Liang HB, He JR, Tu XQ, et al. MicroRNA-140-5p: a novel circulating biomarker for early warning of late-onset post-stroke depression. J Psychiatr Res. 2019;115:129-141. doi:10.1016/j. jpsychires.2019.05.018

14. Liang Z, Jia Y, Li Z, et al. Urinary biomarkers for diagnosing poststroke depression in patients with type 2 diabetes mellitus. Diabetes Metab Syndr Obes. 2019;12:1379-1386. doi:10.2147/ DMSO.S215187

15. Zhang W, Zhang X, Novel Urinary A. Metabolite signature for non-invasive post-stroke depression diagnosis. Cell Biochem Biophys. 2015;72(3):661-667. doi:10.1007/s12013-014-0472-9

16. Xie J, Han Y, Hong Y, et al. Identification of potential metabolite markers for middle-aged patients with post-stroke depression using urine metabolomics. Neuropsychiatr Dis Treat. 2020;16:2017-2024. doi:10.2147/NDT.S271990

17. Geng C, Guo Y, Wang C, et al. Comprehensive evaluation of lipopolysaccharide-induced changes in rats based on metabolomics. J Inflamm Res. 2020;13:477-486. doi:10.2147/JIR.S266012

18. Emmerzaal TL, Preston G, Geenen B, et al. Impaired mitochondrial complex I function as a candidate driver in the biological stress response and a concomitant stress-induced brain metabolic reprogramming in male mice. Transl Psychiatry. 2020;10(1):176. doi:10.1038/s41398-020-0858-y

19. Zheng P, Wang Y, Chen L, et al. Identification and validation of urinary metabolite biomarkers for major depressive disorder. $\mathrm{Mol}$ Cell Proteomics. 2013;12(1):207-214. doi:10.1074/mcp. M112.021816

20. Chen JJ, Xie J, Li WW, et al. Age-specific urinary metabolite signatures and functions in patients with major depressive disorder. Aging (Albany NY). 2019;11(17):6626-6637. doi:10.18632/aging.102133

21. Tang Y, Li Y, Sun J, et al. Selection of an optimal combination panel to better triage COVID-19 hospitalized patients. J Inflamm Res. 2020;13:773-787. doi:10.2147/JIR.S273193
22. Huang Q, Cao Y, Wang S, Zhu R. Creation of a novel inflammation-based score for operable colorectal cancer patients. J Inflamm Res. 2020;13:659-671. doi:10.2147/JIR.S271541

23. Jasmine C, Othman S, Carin L, et al. MetaboAnalyst 4.0: towards more transparent and integrative metabolomics analysis. Nucleic Acids Res. 2018;46(W1):W486-W494. doi:10.1093/nar/gky310

24. Zheng P, Chen JJ, Huang T, et al. A novel urinary metabolite signature for diagnosing major depressive disorder. J Proteome Res. 2013;12(12):5904-5911. doi:10.1021/pr400939q

25. Wu Y, Tang J, Zhou C, et al. Quantitative proteomics analysis of the liver reveals immune regulation and lipid metabolism dysregulation in a mouse model of depression. Behav Brain Res. 2016;311:330-339. doi:10.1016/j.bbr.2016.05.057

26. Rao C, Shi H, Zhou C, et al. Hypothalamic proteomic analysis reveals dysregulation of glutamate balance and energy metabolism in a mouse model of chronic mild stress-induced depression. Neurochem Res. 2016;41(9):2443-2456. doi:10.1007/s11064-0161957-2

27. Wang Z, Li W, Chen J, et al. Proteomic analysis reveals energy metabolic dysfunction and neurogenesis in the prefrontal cortex of a lipopolysaccharide-induced mouse model of depression. Mol Med Rep. 2016;13(2):1813-1820. doi:10.3892/mmr.2015.4741

28. Loubinoux I, Kronenberg G, Endres M, et al. Post-stroke depression: mechanisms, translation and therapy. J Cell Mol Med. 2012;16 (9):1961-1969. doi:10.1111/j.1582-4934.2012.01555.x

29. Levada OA, Troyan AS. Poststroke depression biomarkers: a narrative review. Front Neurol. 2018;9:577. doi:10.3389/ fneur.2018.00577

30. Ramasubbu R, Tobias R, Buchan AM, et al. Serotonin transporter gene promoter region polymorphism associated with poststroke major depression. J Neuropsychiatry Clin Neurosci. 2006;18 (1):96-99. doi:10.1176/jnp.18.1.96

31. Vataja R, Pohjavaara T, Leppavuori A, et al. Magnetic resonance imaging correlates of depression after ischemic stroke. Arch Gen Psychiatry. 2001;58:925-931. doi:10.1001/archpsyc.58.10.925

32. Zheng P, Zeng B, Zhou C, et al. Gut microbiome remodeling induces depressive-like behaviors through a pathway mediated by the host's metabolism. Mol Psychiatry. 2016;21(6):786-796. doi:10.1038/ mp.2016.44

33. Zhang J, Ma L, Chang L, Pu Y, Qu Y, Hashimoto K. A key role of the subdiaphragmatic vagus nerve in the depression-like phenotype and abnormal composition of gut microbiota in mice after lipopolysaccharide administration. Transl Psychiatry. 2020;10(1):186. doi:10.1038/s41398-020-00878-3

34. Chen JJ, He S, Fang L, et al. Age-specific differential changes on gut microbiota composition in patients with major depressive disorder. Aging (Albany NY). 2020;12(3):2764-2776. doi:10.18632/ aging. 102775

35. Chen RL, Balami JS, Esiri MM, Chen LK, Buchan AM. Ischemic stroke in the elderly: an overview of evidence. Nat Rev Neurol. 2010;6(5):256-265. doi:10.1038/nrneurol.2010.36

36. Nóbrega JE, Tonelotto Dos Santos J, Serrano-Mujica LK, et al. Oxidative stress and biochemical markers in prenatally androgenized sheep after neonatal treatment with GnRH agonist. J Inflamm Res. 2019;12:65-71. doi:10.2147/JIR.S190260 


\section{Publish your work in this journal}

Neuropsychiatric Disease and Treatment is an international, peerreviewed journal of clinical therapeutics and pharmacology focusing on concise rapid reporting of clinical or pre-clinical studies on a range of neuropsychiatric and neurological disorders. This journal is indexed on PubMed Central, the 'PsycINFO' database and CAS, and is the official journal of The International Neuropsychiatric Association (INA). The manuscript management system is completely online and includes a very quick and fair peer-review system, which is all easy to use. Visit http://www.dovepress.com/testimonials.php to read real quotes from published authors.

Submit your manuscript here: https://www.dovepress.com/neuropsychiatric-disease-and-treatment-journal 